Обоснование. Учитывая роль висцерального ожирения в развитии мужского бесплодия, актуально исследование показателей качества эякулята, ассоциированных со снижением массы тела.

Цель. Оценка ассоциации показателей качества эякулята и снижения массы тела у пациентов с бесплодием, постпубертатным висцеральным ожирением и неотягощенным андрологическим анамнезом.

Методы. В ретроспективное исследование случай-контроль были включены 33 бесплодных мужчины с постпубертатным алиментарным висцеральным ожирением в возрасте до 30 лет. Всем пациентам были даны рекомендации по снижению массы тела (гипокалорийное питание, ежедневные аэробные физические нагрузки). Проводилась оценка массы тела, индекса массы тела, окружности талии, липидного спектра крови, антиоксидантной активности эякулята, его электронномикроскопическое исследование исходно и в динамике через 6 мес. По окончании исследования пациенты были разделены на 2 группы: 1-я - снизившие массу тела на 5\% и более $(\mathrm{n}=16)$ и 2-я - не достигшие этого результата $(\mathrm{n}=17)$.

Результаты. Исходные значения изучаемых показателей у пациентов двух групп не различались, за исключением массы тела. Через 6 мес изменения всех показателей эякулята (количество сперматозоидов в 1 мл эякулята, доля их морфологически нормальных форм, число сперматозоидов с недостаточно конденсированным хроматином и увеличение антиоксидативной активности эякулята оказались статистически значимо различными. Были выявлены статистически значимые различия в величинах изменений исследуемых показателей за 6 мес у пациентов сравниваемых групп. При этом увеличение количества сперматозоидов в 1 мл эякулята, увеличение содержания их морфологически нормальных форм, уменьшение числа сперматозоидов с недостаточно конденсированным хроматином и увеличение антиоксидативной активности эякулята являлись настолько выраженными, что при отсутствии статистически значимых различий между группами в этих параметрах на момент начала медицинского вмешательства по снижению массы тела, через 6 мес эти изменения привели к тому, что показатели оказались статистически значимо лучше в 1-й, нежели во 2-й группе.

Заключение. У молодых мужчин с постпубертатным висцеральным ожирением и неотягощенным андрологическим анамнезом клинически значимое снижение массы тела ассоциировано с улучшением показателей качества эякулята.

КЛЮЧЕВЫЕ СЛОВА: ожирение; сперматогенез; эякулят.

\title{
SEMEN QUALITY INDICATORS ASSOCIATED WITH REDUCED BODY WEIGHT IN YOUNG INFERTILE MEN WITH PATHOZOOSPERMIA, POSTPUBERTAL VISCERAL OBESITY AND NORMAL ANDROLOGICAL HISTORY
}

(c) Daniil N. Bobkov*, Roman V. Rozhivanov, Larisa V. Savelyeva

Endocrinology Research Centre, Moscow, Russia

BACKGROUND: Considering the negative impact of visceral obesity on fertility, it is important to study semen quality indicators associated with reduced body weight.

AIMS: Evaluation of semen quality indicators associated with reduced body weight in patients with infertility, postpubertal visceral obesity and normal andrological history.

MATERIALS AND METHODS: 33 infertile men under 30 years with post-pubertal and alimentary visceral obesity were included into retrospective case-control study. All patients have been followed through a six month weight loss program (hypocaloric diet, daily aerobic physical activity). The waist circumference, blood lipid levels, seminal antioxidant capacity and scanning electron microscopy analysis were determined before and after weight loss program. At the end of the study, patients were divided into two groups according to weight loss. The first group reduced their body weight by $5 \%$ or more $(n=16)$, second group didn't achieve the goal $(n=17)$.

RESULTS: Statistically significant differences were identified in the ejaculate parameters between patients of two groups. Though at the time of initiation of medical intervention two groups were comparable except for body mass, after six months in the first group the number of sperm in $1 \mathrm{ml}$ ejaculate, morphologically normal forms and the total antioxidant capacity of ejaculate increased while the number of sperm DNA integrity decreased.

CONCLUSION: In young men with postpubertal visceral obesity and normal andrological history clinically significant body weight reduction is associated with improved semen quality indicators.

KEYWORDS: obesity; spermatogenesis; ejaculate. 


\section{ОБОСНОВАНИЕ}

Как в отечественных, так и в зарубежных работах была установлена негативная роль висцерального ожирения в развитии патозооспермии [1-3]. Одной из основных гипотез патогенеза бесплодия является гипотеза о роли избыточной ароматизации тестостерона в жировой ткани [4]. При этом у мужчин с ожирением часто развивается гипогонадизм, связанный с нарушением механизма отрицательной обратной связи гипофиз-гонады [5]. В предлагаемом нами исследовании предполагается оценить показатели качества эякулята, ассоциированные со снижением жировой массы тела у пациентов с патозооспермией в условиях отсутствия в патогенезе бесплодия гипогонадизма.

\section{ЦЕЛЬ}

Оценка показателей качества эякулята, ассоциированных со снижением массы тела у пациентов с бесплодием, постпубертатным висцеральным ожирением и неотягощенным андрологическим анамнезом.

\section{МЕТОДЫ}

\section{Дизайн исследования}

Ретроспективное исследование случай-контроль.

\section{Критерии соответствия}

Критерии включения: совершеннолетние пациенты мужского пола с постпубертатным алиментарным висцеральным (окружность талии от 98 см) ожирением в возрасте до 30 лет включительно с патозооспермией (астено-, тератозооспермия и любые их комбинации, а также нормозооспермия при выявлении недостаточно конденсированного хроматина) из пар, проходивших обследование по поводу бесплодия, наблюдаемого в течение 1 года и более.

Критерии исключения: индекс массы тела (ИМТ) $\geq 35$ кг/м², объем любого из яичек менее 15 мл, нарушения кариотипа, задержка полового развития, наличие в анамнезе крипторхизма, варикоцеле, воспалительных заболеваний, опухолей, травм или хирургических вмешательств на половых органах и области головного мозга, включая гипофиз, олигозооспермия, криптозооспермия, азооспермия, бактериоспермия, лейкоспермия, урогенитальные инфекции, носительство антиспермальных антител, сахарный диабет, гипогонадизм (уровень тестостерона <12 нмоль/л), гипотиреоз, гиперпролактинемия, гиперэстрогенемия, гиперкортицизм, невыполнение программы исследования.

\section{Условия проведения}

Пациенты, проходившие обследование в отделениях андрологии и урологии; терапии эндокринопатий ЛРО ФГБУ «НМИЦ эндокринологии» Минздрава России.

Продолжительность исследования

С октября 2019 г. по апрель 2020 г. Данные собирались двукратно.
Описание медицинского вмешательства

Осуществлялся забор крови в пробирки типа «вакутейнер» в утреннее время натощак из локтевой вены, а также эякулята в стерильные контейнеры путем мастурбации (половое воздержание 3-5 сут).

Всем мужчинам были даны рекомендации по снижению массы тела, включающие гипокалорийное питание, ежедневные аэробные физические нагрузки. Расчет калорийности суточного рациона в килокалориях проводился по формуле, рекомендованной ВО3, с учетом возраста, веса, уровня физической активности мужчин 18-30 лет: $(\mathbf{0 , 0 6 3 0} \times$ вес в кг + 2,8957) $\times \mathbf{2 4 0}$. Поскольку у всех исследуемых уровень физической активности был низким, использовался коэффициент 1. Для снижения массы тела полученную величину уменьшали на 20\%. Минимальная суточная калорийность рациона питания составила 1800 ккал. В случае исходного потребления больными 3000 ккал и более в сутки постепенно уменьшали калорийность суточного рациона на 300-500 ккал в неделю до достижения рассчитанной индивидуальной нормы калорий. Всем пациентам рекомендовали дробный прием пищи (не менее 4 раз в день), так, чтобы ее основной объем приходился на первую половину дня, ужин был калорийностью не более 15-20\% суточной нормы и не позднее чем за 4 ч до сна. Баланс макронутриентов распределяли следующим образом: потребление жиров сокращалось до 25-30\% нормы суточной калорийности, белков - 15-20\%, углеводов - 55-60\%. Пациентам с дислипидемией рекомендовали соблюдение гиполипидемической диеты. Для повышения уровня физической активности всем пациентам рекомендовалась ежедневная ходьба 10000 шагов и умеренные силовые нагрузки по 30-45 мин в день не менее 3 раз в неделю. Длительность вмешательства по снижению массы тела составила 6 мес.

\section{Основной исход исследования}

Основным исходом исследования является получение данных о показателях качества эякулята, ассоциированных со снижением массы тела у пациентов с постпубертатным висцеральным ожирением и неотягощенным андрологическим анамнезом.

\section{Дополнительные исходы исследования}

Дополнительным исходом исследования является выявление метаболических факторов, ассоциированных со снижением массы тела.

\section{Анализ в подгруппах}

Анализ в подгруппах проводился ретроспективно. Поскольку снижение массы тела являлось исходом медицинского вмешательства, подгруппы формировались по этому исходу: снизившие - 1-я группа и не снизившие массу тела - 2-я группа. За снижение массы тела принималась величина, составляющая не менее $5 \%$ исходной массы тела. Выбор данной величины был обусловлен тем, что, по данным различных авторов, для того, чтобы обеспечить метаболические эффекты лечения ожирения, необходимо снизить массу тела не менее чем на 5\% (клинически значимое снижение массы тела) [6-8]. 
Методы регистрации исходов

У всех пациентов были определены ИМТ и окружность талии (ОТ). Концентрацию биохимических показателей сыворотки крови - холестерина (ХC), холестерина липопротеидов низкой плотности (ЛПНП), холестерина липопротеидов высокой плотности (ЛПВП), триглицеридов (ТГ) определяли на биохимическом анализаторе HITACHI (Biohringer Mannheim, Япония). Оценка спермограмм осуществлялась в соответствии с рекомендациями ВОЗ, 2010 г. путем световой микроскопии с помощью микроскопа Olimpus 41 CX (Япония) и камеры Маклера того же производителя [9]. Учитывая, что на параметры спермограммы может влиять множество различных факторов, ее анализировали двукратно (ориентировались на лучший результат). Кроме того, с целью оценки содержания сперматозоидов с интактными головками и отсутствием патологических изменений (норма >4\%), сперматозоидов с недостаточно конденсированным хроматином (норма <30\%), а также оценки патологических изменений головки, шейки, акросомы и жгутика сперматозоидов проводилось электронно-микроскопическое исследование эякулята (ЭМИС). Эякулят после разжижения фиксировали 2,5\% раствором глутарового альдегида на 0,1 М какодилатном буфере (pH 7,2-7,4) и 1\% р-ром осмиевой кислоты и заливали в эпоксидную смолу. Ультратонкие срезы получали на ультрамикротоме Reichert III и просматривали в электронном микроскопе Hitachi 700 [10-12]. Кроме того, по экспериментальной методике определялась антиоксидативная активность (AОА) эякулята с использованием многофункционального потенциометрического анализатора «МПА-1» (НПВП «Ива», г. Екатеринбург) и медиаторной системы, содержащей $\mathrm{K}_{3}\left[\mathrm{Fe}(\mathrm{CN})_{6}\right] / \mathrm{K}_{4}\left[\mathrm{Fe}(\mathrm{CN})_{6}\right]$ в соотношении 0,01 M/0,0001 М. Аликвота эякулята 0,2 мл, буферный раствор с медиаторной системой - 1 мл [13].

\section{Этическая экспертиза}

Исследование было одобрено на заседании ученого совета ФГБУ «Эндокринологический научный центр» Минздрава России (Протокол №1 от 01.02.2017г.).

\section{Статистический анализ}

Принципы расчета размера выборки: исследование «пилотное», размер выборки предварительно не рассчитывался.

Методы статистического анализа данных. Статистическая обработка полученных данных была проведена с использованием пакета прикладных программ Statistica (StatSoft Inc. США, версия 8.0). Сравнение исходных данных обследования двух групп пациентов, величин изменения исследуемых параметров, а также данных обследования, полученных через 6 мес после начала вмешательства по снижению массы тела, осуществлялось с использованием U-критерия Манна-Уитни. Сравнение данных в динамике (исходных и конечных значений) в каждой из групп пациентов - путем теста Вилкоксона. Распределения количественных данных представлены в виде медиан и границ интерквартильного интервала. Базовым уровнем статистической значимости считали 0,05. Применялась поправка Бонферрони на множественные сравнения, в связи с чем при сравнении групп до терапии по 14 показателям пороговый
$\mathrm{P}_{0}=0,0035$, после терапии группы сравнивались по 13 показателям, $\mathrm{P}_{0}=0,0038$.

\section{РЕЗУЛЬТАТЫ}

Объекты (участники) исследования

В исследование были включены 33 совершеннолетних пациента мужского пола с постпубертатным алиментарным висцеральным (ОТ от 98 см) ожирением, патозооспермией (нормозооспермия с нарушенной конденсацией хроматина - 3 пациента, астенозооспермия - 1, астенозооспермия с нарушенной конденсацией хроматина - 14, астенотератозооспермия - 1 , астенотератозооспермия с нарушенной конденсацией хроматина - 14) в возрасте до 30 лет. Через 6 мес, после завершения программы снижения массы тела, пациенты были разделены на 2 подгруппы: 1-я - 16 мужчин со снижением массы тела 5\% и более $(12,0[11,0 ; 14,0] \%)$ и 2-я - 17 мужчин со снижением массы тела менее 5\% $(2,2[1,0 ; 4,1] \%)$.

\section{Основные результаты исследования}

Исходные значения изучаемых показателей пациентов двух групп статистически значимо не различались, кроме массы тела (табл. 1). Через 6 мес у мужчин, снизивших массу тела более чем на 5\%, были отмечены статистически значимые увеличение количества сперматозоидов в 1 мл эякулята, содержание в эякуляте живых сперматозоидов, их морфологически нормальных форм, уменьшение числа сперматозоидов с недостаточно конденсированным хроматином и увеличение АОА эякулята. Эти параметры у пациентов, не снизивших массу тела, статистически значимо не изменились. Были выявлены статистически значимые различия в величинах изменений за 6 мес всех исследуемых показателей эякулята у пациентов сравниваемых групп. При этом увеличение количества сперматозоидов в 1 мл эякулята, увеличение содержания их морфологически нормальных форм, уменьшение числа сперматозоидов с недостаточно конденсированным хроматином и увеличение АОА эякулята являлись настолько выраженными, что при отсутствии статистически значимых различий между группами в этих параметрах на момент начала медицинского вмешательства по снижению массы тела через 6 мес эти изменения привели к тому, что показатели этих параметров оказались статистически значимо лучше в 1-й, нежели во 2-й группе.

Дополнительные результаты исследования

Наряду с улучшением ряда показателей качества эякулята у пациентов со снижением массы тела более 5\% было отмечено статистически значимое уменьшение уровней холестерина и ЛПНП, чего не отмечалось у пациентов 2-й группы. При этом уменьшение уровня холестерина, при отсутствии статистически значимых различий между группами на момент начала исследования, привело к появлению статистически значимых различий через 6 мес от начала вмешательства по снижению массы тела.

Нежелательные явления

Нежелательные явления отсутствовали. 
Таблица 1. Результаты обследования мужчин

\begin{tabular}{|c|c|c|c|}
\hline & $\begin{array}{c}\text { Группа } 1 \\
(n=16)\end{array}$ & $\begin{array}{c}\text { Группа } 2 \\
(n=17)\end{array}$ & $\mathbf{p}^{*}$ \\
\hline Возраст, лет & $29[26 ; 30]$ & $29[28 ; 29]$ & 0,682 \\
\hline 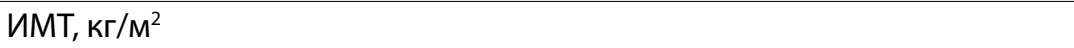 & $29,0[28,1 ; 30,4]$ & $28,7[26,8 ; 30,3]$ & 0,363 \\
\hline ИМТ через 6 мес, кг/м² & $25,1[24,8 ; 26,6]$ & $28,1[26,7 ; 29,3]$ & 0,002 \\
\hline **p & $<0,001$ & 0,002 & \\
\hline Величина изменения ИМТ, кг/м² & $-3,8[-4,3 ;-3,1]$ & $-0,6[-1,1 ;-0,3]$ & $<0,001$ \\
\hline Масса тела, кг & $93[88 ; 96]$ & $85[82 ; 89]$ & 0,010 \\
\hline Масса тела через 6 мес, кг & $81[78 ; 84]$ & $85[80 ; 89]$ & 0,101 \\
\hline${ }^{* *} p$ & $<0,001$ & 0,002 & \\
\hline Величина изменения массы тела, кг & $-12[-14 ;-11]$ & $-2[-4 ;-1]$ & $<0,001$ \\
\hline OT, сM & $102[100 ; 109]$ & $100[99 ; 107]$ & 0,204 \\
\hline ОТ через 6 мес, см & $97[94 ; 102]$ & $98[97 ; 104]$ & 0,136 \\
\hline${ }^{* *} \mathrm{p}$ & $<0,001$ & $<0,001$ & \\
\hline Величина изменения ОТ, см & $-6[-7 ;-5]$ & $-2[-3 ;-1]$ & $<0,001$ \\
\hline ХС, ммоль/л & $5,6[5,4 ; 5,7]$ & $6,0[5,4 ; 6,5]$ & 0,465 \\
\hline ХС через 6 мес, ммоль/л & $4,9[4,7 ; 5,2]$ & $5,6[5,1 ; 6,2]$ & 0,001 \\
\hline${ }^{* *} p$ & $<0,001$ & 0,004 & \\
\hline Величина изменения XС, ммоль/л & $-0,7[-1,0 ;-0,4]$ & $-0,3[-0,6 ; 0,0]$ & 0,007 \\
\hline ХС ЛПНП, ммоль/л & $3,8[3,5 ; 4,4]$ & $4,0[3,2 ; 4,9]$ & 0,901 \\
\hline ХС лПНП через 6 мес, ммоль/л & $3,5[3,4 ; 4,0]$ & $3,9[3,2 ; 4,3]$ & 0,762 \\
\hline${ }^{* *} \mathrm{p}$ & 0,001 & 0,041 & \\
\hline Величина изменения ХС ЛПНП, ммоль/л & $-0,2[-0,2 ;-0,1]$ & $-0,1[-0,3 ; 0,1]$ & 0,231 \\
\hline ХС лПВП, ммоль/л & $0,9[0,9 ; 1,0]$ & $1,0[1,0 ; 1,2]$ & 0,109 \\
\hline ХС ЛПВП через 6 мес, ммоль/л & $0,9[0,9 ; 1,1]$ & $1,2[1,0 ; 1,2]$ & 0,058 \\
\hline${ }^{* *} p$ & 1,0 & 0,551 & \\
\hline Величина изменения ХС ЛПВП, ммоль/л & $0,0[0,0 ; 0,1]$ & $0,0[-0,1 ; 0,2]$ & 0,845 \\
\hline ТГ, ммоль/л & $2,0[1,7 ; 2,4]$ & $2,0[1,8 ; 2,3]$ & 0,986 \\
\hline ТГ через 6 мес, ммоль/л & $1,7[1,5 ; 2,1]$ & $2,0[1,7 ; 2,2]$ & 0,260 \\
\hline${ }^{* *} p$ & 0,006 & 0,706 & \\
\hline Величина изменения ТГ, ммоль/л & $-0,2[-0,4 ;-0,0]$ & $0,0[-0,1 ; 0,1]$ & 0,006 \\
\hline Кол-во сперматозоидов в 1 мл, млн & $35[24 ; 57]$ & $22[17 ; 37]$ & 0,094 \\
\hline Кол-во сперматозоидов в 1 мл через 6 мес, млн & $42[29 ; 66]$ & $22[16 ; 29]$ & 0,002 \\
\hline${ }^{* *} \mathrm{p}$ & 0,001 & 0,030 & \\
\hline Величина изменения кол-ва сперматозоидов в 1 мл, млн & $6[3 ; 10]$ & $-1[-6 ; 0]$ & $<0,001$ \\
\hline Живые сперм-ды, \% & $90[86 ; 92]$ & $86[82 ; 90]$ & 0,191 \\
\hline Живые сперм-ды через 6 мес, \% & $93[90 ; 97]$ & $82[80 ; 93]$ & 0,010 \\
\hline${ }^{* *} \mathrm{p}$ & 0,002 & 0,074 & \\
\hline Величина изменения живых сперматозоидов, \% & $2[1 ; 5]$ & $-2[-4 ; 1]$ & $<0,001$ \\
\hline$(a+b), \%$ & $53[34 ; 69]$ & $32[20 ; 61]$ & 0,217 \\
\hline$(a+b)$ через 6 мес, \% & $57[38 ; 71]$ & $30[18 ; 44]$ & 0,012 \\
\hline${ }^{* *} p$ & 0,006 & 0,115 & \\
\hline Величина изменения $(\mathrm{a}+\mathrm{b}), \%$ & $3[1 ; 6]$ & $-2[-4 ; 1]$ & 0,001 \\
\hline Нормальные формы, \% & $5[3 ; 8]$ & $3[2 ; 8]$ & 0,167 \\
\hline Нормальные формы через 6 мес, \% & $7[4 ; 9]$ & $4[1 ; 5]$ & 0,002 \\
\hline${ }^{* *} p$ & 0,001 & 0,071 & \\
\hline Величина изменения нормальных форм, \% & $1[1 ; 2]$ & $0[-2 ; 0]$ & $<0,001$ \\
\hline АОА эякулята, Мм-экв & $0,9[0,7 ; 1,0]$ & $1,0[0,9 ; 1,2]$ & 0,063 \\
\hline АОА эякулята через 6 мес, Мм-экв & $2,1[2 ; 2,2]$ & $1,0[0,8 ; 1,1]$ & $<0,001$ \\
\hline${ }^{* *} p$ & $<0,001$ & 0,140 & \\
\hline Величина изменения АОА эякулята, Мм-экв & $1,3[1,2 ; 1,4]$ & $-0,1[-0,2 ; 0,1]$ & $<0,001$ \\
\hline Недостаточно конденсированный хроматин, \% & $46[43 ; 65]$ & $38[36 ; 46]$ & 0,058 \\
\hline Недостаточно конденсированный хроматин через 6 мес, \% & $27[23 ; 29]$ & $38[35 ; 49]$ & $<0,001$ \\
\hline${ }^{* *} p$ & $<0,001$ & 0,164 & \\
\hline Величина изменения недостаточно конденсированного хроматина, \% & $-21[-34 ;-15]$ & $1[-1 ; 3]$ & $<0,001$ \\
\hline
\end{tabular}

*U-критерий Манна-Уитни; ** тест Вилкоксона. 


\section{ОБСУЖДЕНИЕ}

\section{Резюме основного результата исследования}

Со снижением жировой массы тела за счет гипокалорийной диеты и аэробной физической активности ассоциировано улучшение показателей качества эякулята при условии, что снижение массы тела является результативным.

\section{Обсуждение основного результата исследования}

Полученные результаты свидетельствуют о возможном благоприятном влиянии снижения массы тела на качество эякулята. Сложно однозначно утверждать о пользе только снижения массы тела пациентов, поскольку оно, как правило, сопряжено и с другими качественными изменениями образа жизни. Тем не менее при клинически значимом снижении массы тела мы отметили улучшение большинства параметров спермограммы. Исходные данные пациентов вряд ли могли повлиять на результат лечения, так как по исходным исследуемым параметрам статистические различия между группами отсутствовали. Однако, поскольку масса тела являлась критерием ретроспективного формирования групп, является целесообразным рассмотреть исходные различия групп (до применения поправки Бонферрони) в отношении этого первичного показателя. Без применения поправки Бонферрони различия между группами в исходной величине массы тела являются статистически значимыми, $\mathrm{p}=0,010$. Но эти различия не искажают сделанных заключений, так как та группа, которая продемонстрировала наилучшие результаты как в отношении снижения массы тела, так и в отношении показателей качества эякулята, имела исходно большую величину массы тела (ожирение было более выраженным) по сравнению с группой пациентов, которые продемонстрировали в динамике худшие результаты. Наличие более выраженного ожирения на момент начала лечения у пациентов с хорошим результатом дополнительно подтверждает положительное влияние снижения массы тела на показатели качества эякулята. Ожидаемо, что наряду с хорошим снижением массы тела, у пациентов 1й группы улучшился липидный спектр крови и АОА эякулята. При этом маловероятно, что дислипидемия действует на сперматогенез напрямую, но она может быть ассоциирована с оксидативным стрессом [14]. В ряде работ было установлено, что оксидативный стресс может являться патогенетическим фактором патозооспермии [15-17].

Полученные нами результаты согласуются с данными других исследователей. Так, группа пациентов с наибольшей потерей веса при соблюдении гипокалорий- ной диеты имели статистически значимое увеличение сперматозоидов с нормальной морфологией, общего количества сперматозоидов и уменьшения фрагментации ДНК $[18,19]$. В другом исследовании оценивали влияние бариатрической хирургии - через год после проведения операции было отмечено увеличение концентрации сперматозоидов, которое являлось статистически значимым у мужчин с исходной патозооспермией [20]. В некоторых работах при улучшении антропометрических показателей значимо уменьшались уровни маркеров оксидативного стресса: данные показаны в серии клинических случаев, где при уменьшении абдоминального жира улучшались целостность ДНК сперматозоидов и исходы беременности, а также снижалось количество поврежденных белков в результате оксидативного стресса и увеличивался уровень супероксиддисмутазы $2[5,21]$.

\section{Ограничения исследования}

Наряду со снижением массы тела благоприятно сказаться на качестве эякулята могли и другие позитивные факторы изменения образа жизни пациентов. Также ограничениями являются: малый размер выборки, ретроспективное деление пациентов на группы и валидность метода оценки АОА эякулята, так как методика является экспериментальной и требует проведения дальнейших исследований.

\section{ЗАКЛЮЧЕНИЕ}

У молодых мужчин с постпубертатным висцеральным ожирением и неотягощенным андрологическим анамнезом клинически значимое снижение массы тела ассоциировано с улучшением показателей качества эякулята.

\section{ДОПОЛНИТЕЛЬНАЯ ИНФОРМАЦИЯ}

Источники финансирования. Работа выполнена на базе ФГБУ «НМИЦ эндокринологии» Минздрава России.

Конфликт интересов. Авторы декларируют отсутствие явных и потенциальных конфликтов интересов, связанных с содержанием настоящей статьи.

Участие авторов. Бобков Д.Н. - набор материала, обработка полученных данных, написание статьи; Роживанов Р.В. - разработка концепции исследования, набор материала, редактирование текста статьи; Савельева Л.В. - набор материала, редактирование текста статьи. Все авторы одобрили финальную версию статьи перед публикацией, выразили согласие нести ответственность за все аспекты работы, подразумевающую надлежащее изучение и решение вопросов, связанных с точностью или добросовестностью любой части работы.

\section{СПИСОК ЛИТЕРАТУРЫ | REFERENCES}

1. Роживанов Р.В., Курбатов Д.Г. Структура патозооспермии у молодых мужчин с постпубертатным висцеральным ожирением и неотягощенным андрологическим анамнезом // Ожирение и метаболизм. — 2017. — Т.14. — №4. - C. 32-37. [Rozhivanov RV., Kurbatov DG. The structure of pathozoospermia in young men with post-pubertal visceral obesity and a non-aggravated andrologic anamnesis. Obesity and Metabolism. 2017;14(4):32-37. (In Russ.)] doi: https://doi.org/10.14341/omet2017432-37

2. Campbell JM, Lane M, Owens JA, Bakos HW. Paternal obesity negatively affects male fertility and assisted reproduction outcomes: a systematic review and metaanalysis. Reprod Biomed Online. 2015;31(5):593-604. doi: https://doi.org/10.1016/j.rbmo.2015.07.012

3. Faure C, Dupont C, Baraibar MA, et al. In Subfertile Couple, Abdominal Fat Loss in Men Is Associated with Improvement of Sperm Quality and Pregnancy: A Case-Series. PLoS One. 2014;9(2):e86300. doi: https://doi.org/10.1371/journal.pone.0086300

4. Xu X, Sun M, Ye J, et al. The Effect of Aromatase on the Reproductive Function of Obese Males. Horm Metab Res. 2017;49(08):572-579. doi: https://doi.org/10.1055/s-0043-107835 
5. Grossmann M. Hypogonadism and male obesity: Focus on unresolved questions. Clin Endocrinol (Oxf). 2018;89(1):11-21. doi: https://doi.org/10.1111/cen.13723

6. Jensen MD, Ryan DH, Apovian CM, et al. 2013 AHA/ACC/ TOS Guideline for the Management of Overweight and Obesity in Adults. Circulation. 2014;129(25 suppl 2):S102-S138 doi: https://doi.org/10.1161/01.cir.0000437739.71477.ee

7. Williamson DA, Bray GA, Ryan DH. Is $5 \%$ weight loss a satisfactory criterion to define clinically significant weight loss? Obesity. 2015;23(12):2319-2320. doi: https://doi.org/10.1002/oby.21358

8. Дедов И.И., Мельниченко Г.А., Шестакова М.В., и др. Национальные клинические рекомендации по лечению морбидного ожирения у взрослых. 3-ий пересмотр (лечение морбидного ожирения у взрослых) // Ожирение и метаболизм. 2018 - T.15. - №1. - C. 53-70. [Dedov II, Mel'nichenko GA, Shestakova MV, et al. Russian national clinical recommendations for morbid obesity treatment in adults. 3rd revision (Morbid obesity treatment in adults). Obesity and Metabolism. 2018;15(1):53-70. doi: https://doi.org/10.14341/omet2018153-70

9. World Health Organization Department of Reproductive Health and Research. WHO laboratory manual for the examination and processing of human semen, fifth edition. WHO 2010. Available at: https://www.who.int/reproductivehealth/publications/ infertility/9789241547789/en/

10. Х Хаят С.Ш., Брагина Е.Е., Курило Л.Ф. Ультраструктурное исследование сперматозоидов у пациентов с астенозооспермией. Андрология и генитальная хирургия. — 2012. — Т.13. — №4. —C. 54-61 [Khayat SS, Bragina EE, Kurilo LF. Ultrastructural investigation of human sperm from asthenozoospermic men. Andrologiya igenital'naya khirurgiya. 2012;13(4):54-61. (In Russ.)] doi: https://doi.org/10.17650/2070-9781-2012-4-54-61

11. Брагина Е.Е., Бочарова Е.Н. Количественное электронномикроскопическое исследование сперматозоидов при диагностике мужского бесплодия. Андрология и генитальная хирургия. — 2014. - Т.15. — №1. C. 41-50. [Bragina YY, Bocharova YN. Quantitative electron microscopic examination of sperm for male infertility diagnosis. Andrologiya i genital'naya khirurgiya. 2014;15(1):41-50. (In Russ.)] doi: https://doi.org/10.17650/2070-9781-2014-1-41-50

12. Брагина Е.Е., Замятнина В.А., Бочарова Е.Н., и др. Количественное ультраструктурное исследование хроматина сперматозоидов при нарушении фертильности // Андрология и генитальная хирургия. - 2009. - Т.10. — №1. - С. 44-49. [Bragina EE, Zamyatnina VA, Bocharova EN, et al. Quantitative ultrastructural research research of spermatozoon from patients with fertility infringement. Andrologiya i genital'naya khirurgiya. 2009;10(1):44-49. (In Russ.)]

13. Герасимова Е.Л. Потенциометрия в исследовании антиоксидантной активности биологических объектов. Дис:: канд. хим. наук. - Казань, 2010. [Gerasimova E.L. Potenciometriya $v$ issledovanii antioksidantnoj aktivnosti biologicheskih obyektov. [dissertation] Kazan; 2010. (In Russ).]

14. Koppers AJ, Garg ML, Aitken RJ. Stimulation of mitochondrial reactive oxygen species production by unesterified, unsaturated fatty acids in defective human spermatozoa. Free Radic Biol Med. 2010;48(1):112-119. doi: https://doi.org/10.1016/j.freeradbiomed.2009.10.033

15. Giulini S, Sblendorio V, Xella S, et al. Seminal plasma total antioxidant capacity and semen parameters in patients with varicocele. Reprod Biomed Online. 2009;18(5):617-621. doi: https://doi.org/10.1016/S1472-6483(10)60004-1

16. Roychoudhury S, Sharma R, Sikka S, Agarwal A. Diagnostic application of total antioxidant capacity in seminal plasma to assess oxidative stress in male factor infertility. J Assist Reprod Genet. 2016;33(5):627-635. doi: https://doi.org/10.1007/s10815-016-0677-5

17. Subramanian $V$, Ravichandran A, Thiagarajan $N$, et al. Seminal reactive oxygen species and total antioxidant capacity: Correlations with sperm parameters and impact on male infertility. Clin Exp Reprod Med. 2018;45(2):88-93. doi: https://doi.org/10.5653/cerm.2018.45.2.88

18. Håkonsen LB, Thulstrup AM, Aggerholm AS, et al. Does weight loss improve semen quality and reproductive hormones? results from a cohort of severely obese men. Reprod Health. 2011;8(1):24. doi: https://doi.org/10.1186/1742-4755-8-24

19. Mir J, Franken D, Andrabi SW, Ashraf M, Rao K. Impact of weight loss on sperm DNA integrity in obese men. Andrologia. 2018;50(4):e12957. doi: https://doi.org/10.1111/and.12957

20. El Bardisi H, Majzoub A, Arafa M, et al. Effect of bariatric surgery on semen parameters and sex hormone concentrations: a prospective study. Reprod Biomed Online. 2016;33(5):606-611. doi: https://doi.org/10.1016/j.rbmo.2016.08.008

21. 1Gutiérrez L, García JR, Rincón M de J, et al. Efecto de una dieta hipocalórica en el estrés oxidativo en sujetos obesos sin prescripción de ejercicio y antioxidantes. Med Clin (Barc). 2015;145(1):1-6. doi: https://doi.org/10.1016/j.medcli.2013.12.015

\section{ИНФОРМАЦИЯ ОБ АВТОРАХ [AUTHORS INFO]:}

*Бобков Даниил Николаевич, аспирант [Daniil N. Bobkov, MD, postgraduate student]; адрес: Россия, 117036, Москва, ул. Дм. Ульянова, д. 11 [address: 11 Dm. Ulyanova street, 117036, Moscow, Russia]; ORCID: https://orcid.org/0000-0002-7145-8954; eLibrary SPIN: 5311-5050; e-mail: danil-0000@mail.ru

Роживанов Роман Викторович, д.м.н. [Roman V. Rozhivanov, MD, PhD]; ORCID: https://orcid.org/0000-0002-5386-4289; eLibrary SPIN: 8052-3310; e-mail: rrozhivanov@mail.ru

Савельева Лариса Викторовна, к.м.н. [Larisa V. Savelyeva, MD, PhD]; ORCID: https://orcid.org/0000-0002-2808-4846; eLibrary SPIN: 1452-8793; e-mail: slv63@mail.ru

*Автор, ответственный за переписку / Corresponding author.

\section{ЦИТИРОВАТЬ:}

Бобков Д.Н., Роживанов Р.В., Савельева Л.В. Показатели качества эякулята, ассоциированные со снижением массы тела у молодых бесплодных мужчин с патозооспермией, постпубертатным висцеральным ожирением и неотягощенным андрологическим анамнезом // Ожирение и метаболизм. — 2020. — Т. 17. — №4. — С. $340-345$. doi: https://doi.org/10.14341/omet12679

\section{TO CITE THIS ARTICLE:}

Bobkov DN, Rozhivanov RV, Savelyeva LV. Semen quality indicators associated with reduced body weight in young infertile men with pathozoospermia, postpubertal visceral obesity and normal andrological history. Obesity and metabolism. 2020;17(4):340-345. doi: https://doi.org/10.14341/omet12679 